\title{
ANALYTICAL HIERARCHICAL PROCESS BASED SYSTEM FOR IMAGE FUSION
}

\author{
Ashok Kumar ${ }^{1}$, Amruta Shelar ${ }^{1}$, Jim Etheredge ${ }^{1}$, Sandhya Prakash $^{2}$, Sudhansh \\ Sharma $^{2}$ and MP Mishra ${ }^{2}$ \\ ${ }^{1}$ University of Louisiana at Lafayette, LA, USA \\ ${ }^{2}$ IGNOU, India
}

\begin{abstract}
Image fusion has been rapidly gaining importance in application areas such as medical imaging, remote sensing, robotics, navigation and the military. This has led to emergence of several algorithms for image fusion for generating more precise images. This paper presents a novel approach of selecting best image fusion algorithm for a given scenario using Analytical Hierarchical Process (AHP) involving eigen vectors. Evaluations of the proposed approach indicate higher quality of fused images.
\end{abstract}

\section{INTRODUCTION}

Rapid increase in demand for image fusion technology in various research and industrial areas has led to the development of various algorithms and methodologies for implementing it. Huge amount of variation in the information contained in an image and its applicable types of domain have given rise to diverse options for image fusion techniques. It has become a difficult task to manually select an image fusion technique, given varied set images and resources. There is no guarantee that an algorithm selected manually is an excellent fit for a given scenario.

As a solution to this problem, there is a need for automating this process of algorithm selection since the selected algorithm depends on various criteria that user requires depending on domain of image fusion applications. Analysis of each criteria and assigning weight/priority to it is necessary to create a clearer picture of which alternatives fits best for given set of conditions. This analysis is then extended. Such analysis includes building a model that computes and stores the ranking for the image fusion techniques based on different criteria/agents image, sensor, application, and resources. This ranking is computed for each image fusion technique using AHP. AHP uses eigenvectors which are computed iteratively to evaluate ranking based on various evaluation criteria.

\section{RELATED WORK AND LITERATURE SURVEY}

There are various areas where AHP has been used widely. A few of these applications are mentioned here with their goal and brief descriptions. AHP [12] was utilized efficiently in placement of remote terminal units wherein decision making for optimal location was done based on various weighted criteria such as load types, capacity, substation accessibility and grid topology. 
Signal \& Image Processing : An International Journal (SIPIJ) Vol.6, No.5, October 2015

Haibin and Haiwen [14] studied monitoring and diagnosis evaluation of electric apparatus control system. Using AHP, a hierarchical architecture was formed and an analysis was done on electric apparatus control system based on various agents like system characteristics, node agent, subsystem agent and management agent.

In academia, there is a significant amount of work done using AH P. A framework of integrated decision support system [13] was designed for students' project evaluation. That research proposed a framework for group decision support system based on weighted model, and AHP. An AHP based decision model [26] was designed for assessment of Ph.D. quality in universities wherein influencing parameters for assessment are identified and analyzed. They then put to use by AHP to help narrow the gap between qualities for different grades of universities.

An AHP based requirement prioritization model [16] was built for market driven products. It has assisted software engineers in assigning priorities to subsets of requirements that are more important than others. Due to the great efficiency provided by AHP, product manufacturers get a better idea about planning and organizing its product line and eased decision making process for requirements should be included in the product release in the market.

Another area in which AHP turned out to be of great use is knowledge management (KM) implementation [15]. Various decision making processes in a KM implementation are automated due to AHP with the help of KM variables which can be identified for successful implementation of KM.

Various efforts were made in image fusion field to resolve the problem of selection. One of them included multi-agent based Intelligent system [17] for image fusion which made use of JADE for getting hold of agents in the system. These agents determined the ranking of the algorithms and ensured selection of the best fusion algorithm.

There is also a significant amount of work done in similar area where image feature fusion [18] is done using Multi-Attribute Decision Making (MADM). This research proposes that features of image are fused during image retrieval and subjective and objective weighing analysis is done by MADM while final results are derived by TOPSIS.

\section{PROPOSED AHP-BASED MODEL}

The proposed model consists of a process that computes best option in list of options of image fusion techniques for a set of characteristics/criteria. This best option, chosen for a set of characteristics, is then stored in the knowledge base. Whenever computation is done for a set of characteristics, the knowledge base stores results and adaptively learns with every new computation. The computation of the best image fusion technique is done using AHP. AHP consists of a pair wise comparison of image fusion techniques against characteristic/criteria. Input to this process is the relative importance of one set of characteristics over other and one alternative over the other in terms of each characteristic/criteria. This is then transformed into pair-wise comparison matrices. These matrices use eigenvector computation to obtain ranking of image fusion techniques.

The system gets input from user and checks the knowledge base for any computed result. If it finds a desired result set, the knowledge base returns prediction to the user. If not, the knowledge 
Signal \& Image Processing : An International Journal (SIPIJ) Vol.6, No.5, October 2015

base returns a query to AHP process which computes the new result and stores it in the knowledge base. Figure 1 shows the basic architecture of the AHP model.

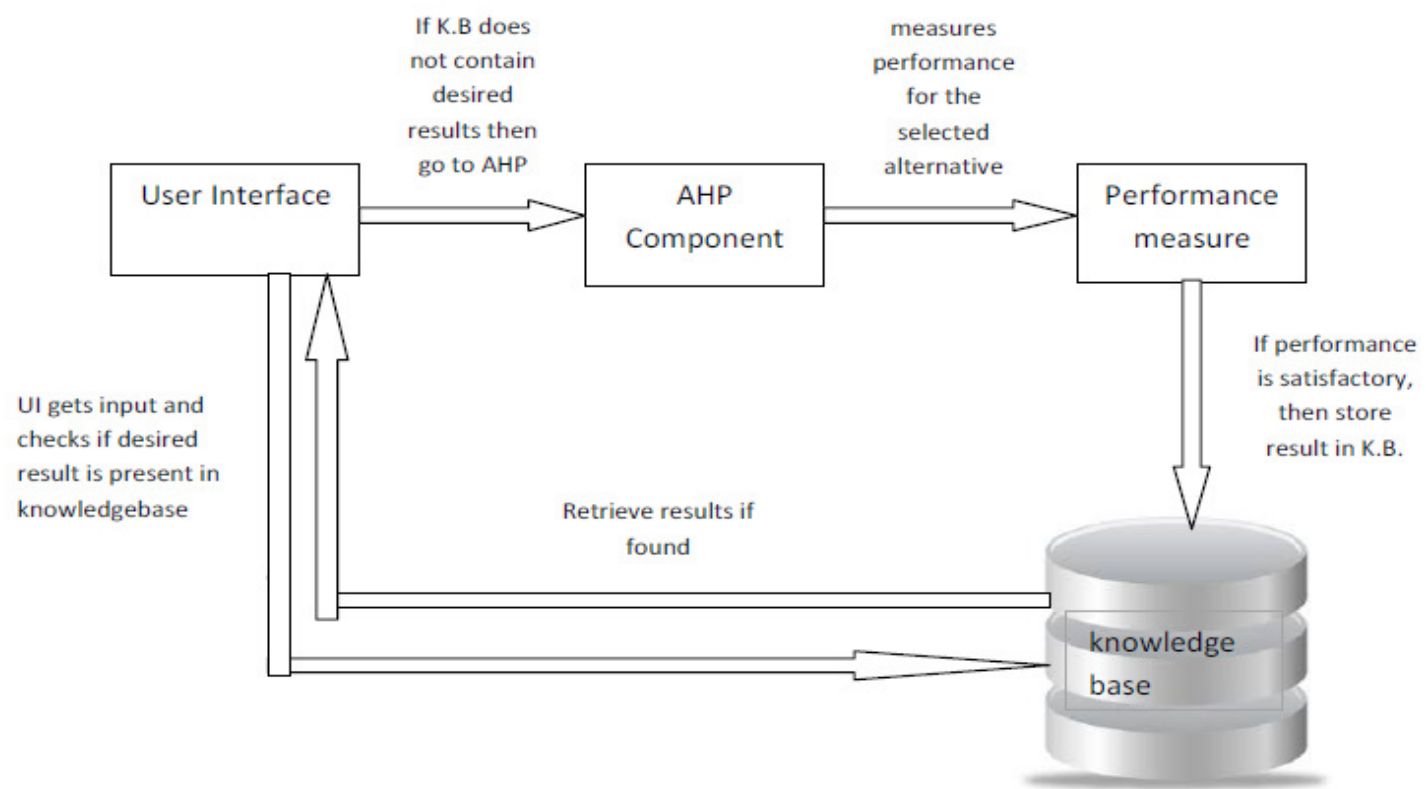

Fig 1. Architecture of AHP based model

\subsection{AHP Component}

AHP selects one of the options of a list of choices. Each choice has a few criteria/parameters attached to it and we can set the weights/priority of each criteria/parameters and have AHP pick the best choice from the list of choices. AHP was introduced by Thomas Saaty [1] in 1970s. It is multi-criteria decision making approach that makes use of eigenvectors to determine best results. AHP is represented in the form of a hierarchical tree with its goal/objective on top most level. Second level is the criteria and sub-criteria. The last level is the alternatives that are associated with each criteria or sub-criteria. Figure 2 represents our proposed formulation.

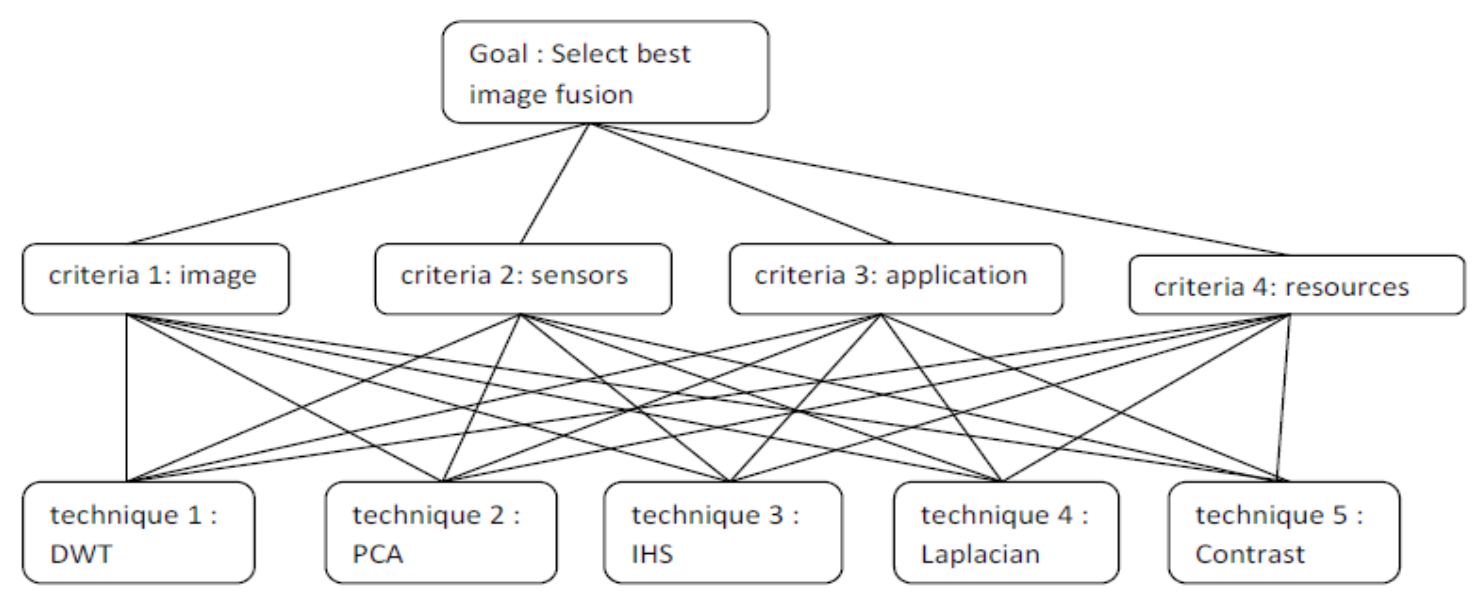

Fig.2 Hierarchical structure of AHP 
Signal \& Image Processing : An International Journal (SIPIJ) Vol.6, No.5, October 2015

\subsection{Working of AHP}

AHP works in three phases [10]. The relative importance score for each criteria or alternatives can be obtained from ranks/importance/preferences that are available. The computation of relative importance uses an AHP scale described in Table 1.

Also, if ranking for the alternatives is available then one can make use of this formula :

$\mathrm{C}_{\mathrm{ij}}=\left[\left(\mathrm{r}_{\mathrm{i}}-\mathrm{r}_{\mathrm{j}}\right) /\left(\mathrm{r}_{\max }-\mathrm{r}_{\min }\right)\right]^{* 9}$ if $\mathrm{r}_{\mathrm{i}}>\mathrm{r}_{\mathrm{j}}$

if $r_{i}>r_{j}$

then $\mathrm{C}_{\mathrm{ji}}=1 / \mathrm{C}_{\mathrm{ij}}$

where $\mathrm{C}_{\mathrm{ij}}, \mathrm{C}_{\mathrm{ji}}$ are the relative importance of $\mathrm{i}^{\text {th }}$ and $\mathrm{j}^{\text {th }}$ criteria in pair-wise comparison matrix while $r_{i}$ and $r_{j}$ are rankings available for criteria $i$ and $j$.

Table 1. The fundamental scale of absolute numbers[7]

\begin{tabular}{|c||c|c|}
\hline $\begin{array}{c}\mathrm{a}_{\mathrm{ij}} \text { (Intensity } \\
\text { of } \\
\text { importance) }\end{array}$ & Condition & Explanation \\
\hline \hline 1 & If $\mathrm{C}_{\mathrm{i}}$ is equally important as $\mathrm{C}_{\mathrm{j}}$ & $\begin{array}{c}\text { Two activities contribute equally to the } \\
\text { objective }\end{array}$ \\
\hline \hline 3 & If $\mathrm{C}_{\mathrm{i}}$ is weakly more important than $\mathrm{C}_{\mathrm{j}}$ & $\begin{array}{c}\text { Experience and judgment slightly favor one } \\
\text { activity over another }\end{array}$ \\
\hline \hline 7 & If $\mathrm{C}_{\mathrm{i}}$ is strongly more important than $\mathrm{C}_{\mathrm{j}}$ & $\begin{array}{c}\text { Experience and judgment strongly favor } \\
\text { one activity over another }\end{array}$ \\
\hline \hline 9 & If $\mathrm{C}_{\mathrm{i}}$ is very strongly more important than $\mathrm{C}_{\mathrm{j}}$ & $\begin{array}{c}\text { An activity is strongly favored and its } \\
\text { dominance demonstrated in practice }\end{array}$ \\
\hline \hline $2,4,6,8$ & If $\mathrm{C}_{\mathrm{i}}$ is absolutely more important than $\mathrm{C}_{\mathrm{j}}$ & $\begin{array}{c}\text { An activity is strongly favored and its } \\
\text { dominance demonstrated in practice }\end{array}$ \\
\hline \hline $1 / 3$ & Intermediate values between the two adjacent & When compromise is needed \\
\hline \hline $1 / 5$ & judgments & \\
\hline \hline $1 / 7$ & If $\mathrm{C}_{\mathrm{i}}$ is weakly less important than $\mathrm{C}_{\mathrm{j}}$ & \\
\hline \hline $1 / 9$ & If $\mathrm{C}_{\mathrm{i}}$ is very strongly less important than $\mathrm{C}_{\mathrm{j}}$ & \\
\hline & If $\mathrm{C}_{\mathrm{i}}$ is absolutely less important than $\mathrm{C}_{\mathrm{j}}$ & \\
\hline
\end{tabular}

Phase 1: Obtaining criteria/agent scores

The pair-wise matrix is computed after the preference and importance of the criteria(aij) is received. This is done by placing computed aij in the right place of pair-wise matrix. This followed by eigenvector computation on pair-wise comparison matrix to obtain relative importance scores which decides ranking/priorities of each criterion to be considered while 
Signal \& Image Processing : An International Journal (SIPIJ) Vol.6, No.5, October 2015

selective best alternative. AHP is proven to give best results when eigenvector computation is used to calculate relative importance.

Eigenvector is computed in following steps as explained in[11]:

step 1 : Given a square matrix (pair-wise comparison matrix) $\mathrm{P}$, we first find its eigenvalues. we find the values of $\lambda$ which satisfy the characteristic equation of the matrix $P$, namely those values of $\lambda$ for which

$$
\operatorname{det}(\mathrm{P}-\lambda \mathrm{I})=0
$$

where $\mathrm{I}$ is the $3 \times 3$ identity matrix.

step 2 : Once the eigenvalues of a matrix $(\mathrm{P})$ have been found, we can find the eigenvectors by Gaussian Elimination.

step 2 (i): For each eigenvalue $\lambda$, we have

$$
(P-\lambda I) x=0,
$$

where $\mathrm{x}$ is the eigenvector associated with eigenvalue $\lambda$.

step 2 (ii) : Find $\mathrm{x}$ by Gaussian elimination. That is, converting the augmented matrix

$$
(\mathrm{P}-\lambda \mathrm{I} \vdots 0)
$$

to row echelon form, and solving the resulting linear system by back substitution to find the eigenvectors associated with each of the eigenvalues.

\section{Phase 2: Obtaining alternatives/image fusion techniques scores}

Similarly, the pair-wise comparison matrices are obtained for all alternatives in terms of each criterion. The eigenvector is computed for alternatives for each criterion. Thus, Eigenvector computations for all alternatives will take place as many times as the number of criteria considered to select the best alternative.

\section{Phase 3: Aggregating criteria scores and alternatives scores to obtain final result}

After obtaining eigenvectors for alternatives, the matrix is formed mapping each alternative eigenvector score to every other criteria by concatenating eigenvectors of all alternatives in terms of all criteria. The matrix formed previously is multiplied with the eigenvector of criteria to get an aggregate score. This aggregate score determines the final ranking of each alternative / algorithm. The higher the aggregate score, the better ranking the alternative/algorithm has.

\subsection{Knowledge base}

When the user inputs a set of characteristics for which best image fusion technique is chosen, the knowledge base accepts that set of characteristics and checks if there is a previously computed result for this set of characteristics. If it finds the desired result, it is retrieved and displayed. If it 
Signal \& Image Processing : An International Journal (SIPIJ) Vol.6, No.5, October 2015

cannot find desired result, the query goes to AHP component where the computation of best alternative/image fusion technique is done and stored back in knowledge base. In this manner with every new computation, the knowledge base adaptively learns best alternatives for most sets of characteristics that user inputs. This helps reduce response time and produces a huge increase in accuracy of the system.

\subsection{Image Fusion Methodologies}

There is a large number of image fusion algorithms available. Only a few of them are used in this approach that covers most domains of image fusion applications. A brief description of these techniques is given next:

\section{A. Intensity-hue-saturation :}

The hue and saturation components consist of spectral information.

The intensity component consists of the spatial information. The Red, Green and Blue channels are first converted to intensity, hue and saturation components. Then the matching of the histogram of the image is done with its intensity component. This method makes sure that spectral information is not disturbed while adding a high amount of spatial information [19][20]. This is followed by converting theIHS channel to the RGB channel.

\section{B. Principle Component Analysis:}

Principle Component Analysis is a spatial domain approach whose function is reduction of multidimension data sets into lower dimensional data sets for the purpose of analysis. It is highly quantitative method that does calculations based on eigenvectors computed from source image matrices thus evaluating weights of each source image from eigenvalues of its covariance [20][21][22][23].

\section{Discrete Wavelet Transform:}

Discrete Wavelet Transform are decomposition tool for multi-resolution image that has various channels representing the image feature on a multi-scale level. It is done based on different frequency sub-bands. DWT converts an image from the spatial to the frequency domain. In this method, decomposition of image takes place by dividing image into horizontal and vertical lines and fusion takes place at each decomposition level. This is then followed by inverse DWT that is carried out on each fused image at all decomposition levels [22][23].

\section{Pyramid based:}

Pyramid based method works with pyramid transforms i.e. it constructs the pyramid transform of the fused image from the pyramid transforms of the source images. This is then followed by reconstruction of the fused image by taking inverse pyramid transform. Pyramid transforms consist of three phases. First, decomposition of is done at different pre-defined levels. Then, phase two fuses or merges input images after decomposition. The resultant matrix becomes the input to recomposition process. Phase three is recomposition i.e. the resultant fused image is developed from the pyramids formed at each level of decomposition[20]. 
Signal \& Image Processing : An International Journal (SIPIJ) Vol.6, No.5, October 2015

There can be various pyramids during fusion. Our approach takes into consideration only two of them: Laplacian pyramid and Contrast pyramid. Laplacian pyramid consist of few steps of decomposition and recomposition. It is done by filtering input images with a predefined low pass filter. This is followed by subtracting input images forming pyramid and decimating input image matrices.

\subsection{Quality Assessment}

A. Mutual Information (MI): MI is defined by the Kullback-Leibler measure[17] which quantifies the degree of dependence between two variables A and B:

$$
I_{A, B}(a, b)=\sum_{x, y} P_{A B}(\mathrm{a}, \mathrm{b}) \log \left[\mathrm{P}_{\mathrm{AB}}(\mathrm{a}, \mathrm{b}) / \mathrm{P}_{\mathrm{A}}(\mathrm{a}) \mathrm{P}_{\mathrm{B}}(\mathrm{b})\right]
$$

where $P_{A}(a) P_{B}(b)$ is the probability associated with the case of complete independence and $P_{A B}(a, b)$ is the joint ('a' intersection 'b') probability. The Mutual Information (MI) between two images is computed using following formula:

$$
\mathrm{M}_{F}^{A B}=\mathrm{I}_{\mathrm{FA}}(\mathrm{f}, \mathrm{a})+\mathrm{I}_{\mathrm{FB}}(\mathrm{f}, \mathrm{b})
$$

B. Visual Information Fidelity : VIF is used to compute difference between test image and reference image provided. It uses HVS model. This is measure for the information that could ideally be extracted by the brain from the reference image[17][24]. Using the HVS model the test and reference images are defined as: The HVS model is used to define the test and reference images as follows :

$$
\begin{gathered}
E=C+N(\text { reference image }) \\
F=D+N^{\prime}(\text { test image })
\end{gathered}
$$

where $E$ and $F$ are the output visual signals of the HVS model and $N$ and $N^{\prime}$ are the HVS noise. The VIF over $\mathrm{N}$ elements is computed as follows :

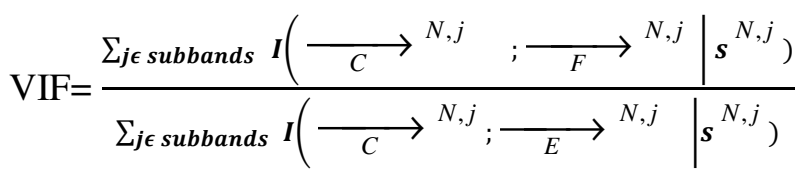

C. Petrovic measure : This quality metric introduced by Xydeas and Petrovic [25]focuses on important visual information associated with edge information of every pixel in the image. A Sobel edge operator is applied to obtain the edge strength $g(n, m)$ and orientation $a(n, m)$ information for each pixel $\mathrm{p}(\mathrm{n}, \mathrm{m})$. Petrovic measure is calculated as follows :

$$
\mathrm{Q}_{P}^{A B / F}(\mathrm{n}, \mathrm{m})=\frac{\sum_{n=\mathbf{1}}^{N} \sum_{\boldsymbol{m}=\mathbf{1}}^{M} \boldsymbol{Q}^{A F}(\boldsymbol{n}, \boldsymbol{m}) \boldsymbol{W}_{A} \boldsymbol{A}(\boldsymbol{n}, \boldsymbol{m})+\boldsymbol{Q}^{B F}(\boldsymbol{n}, \boldsymbol{m}) \boldsymbol{W}_{B}(\boldsymbol{n}, \boldsymbol{m})}{\sum_{n=\mathbf{1}}^{N} \sum_{\boldsymbol{m}=\mathbf{1}}^{M} \boldsymbol{W}_{A}(\boldsymbol{n}, \boldsymbol{m})+\boldsymbol{W}_{B}(\boldsymbol{n}, \boldsymbol{m})}
$$


Signal \& Image Processing : An International Journal (SIPIJ) Vol.6, No.5, October 2015

where_$Q^{A F}(n, m)$ and $Q^{B F}(n, m)$ represent edge preservation values , $W_{A} A(n, m)$ and $W_{B}(n, m)$ are associated weights associated with each edge preservation values.

\subsection{Evaluation}

An initial evaluation of this approach is done using JAVA and MATLAB along with knowledge base in form of a database. MATLAB was chosen because it is a convenient option for core programming of AHP and image fusion algorithms due to its flexibility and simplicity of matrix calculations than any other programming languages. Controls and interfacing are developed using JAVA along with knowledge base in form of MySQL database.

When user inputs priorities for each criterion, the system checks the knowledge base for given user priorities. If desired results are found, it is displayed. If desired result does not exist in knowledge base, it means AHP computation has never been performed for given set of inputs. This leads to sending controls and input to AHP component which comes up with its result set. This result set is verified with the help of performance measure using VIF, MI and Petrovic measure. If results are verified using performance measure and if they are satisfactory, they are stored into knowledge base as results for given set of input criteria priorities. If results could not be verified, the control goes back to performance measure component to check performance measure for next higher ranked alternative in the list of result sets. It keeps running in loop until it finds better ranking alternative yielding acceptable performance.

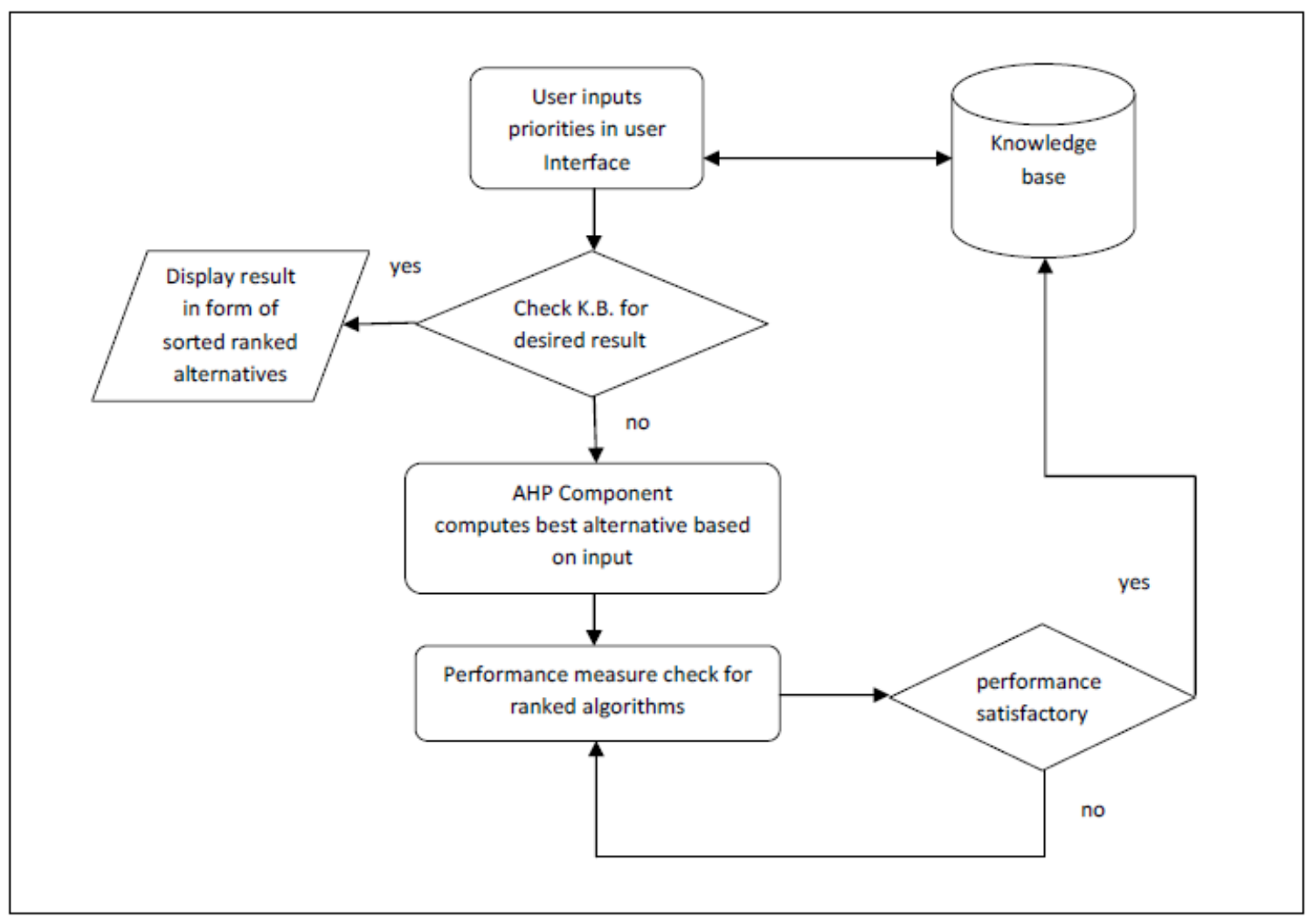

Fig 3. Flowchart for the proposed framework 
Signal \& Image Processing : An International Journal (SIPIJ) Vol.6, No.5, October 2015

\subsection{Illustration and Result Analysis}

We consider four criteria: image, sensor, application, resources. User inputs that image criteria is weakly less important than application while strongly less important than resources. Also, application is strongly important than resources, very strongly more important than sensors. Sensor is weakly less important than image. For the example explained next, the input image is a remote sensing image.

\section{$\underline{\text { Pair-wise matrix for criteria }}$}

\begin{tabular}{|l|l|l|l|l|}
\hline & image & sensors & application & resources \\
\hline image & 1 & 3 & $1 / 3$ & $1 / 5$ \\
\hline sensors & $1 / 3$ & 1 & $1 / 7$ & 3 \\
\hline application & 3 & 7 & 1 & 5 \\
\hline resources & 5 & $1 / 3$ & $1 / 5$ & 1 \\
\hline
\end{tabular}

which can be represented in form of matrix as

$\left[\begin{array}{cccc}1 & 3 & 1 & 0.20 \\ 0.33 & 1 & 0.14 & 3 \\ 1 & 7 & 1 & 5 \\ 5 & 0.33 & 0.20 & 1\end{array}\right]$

Computing normalized eigenvector for above pair wise matrix we get,

$$
\left[\begin{array}{l}
0.1865 \\
0.1500 \\
0.4521 \\
0.2114
\end{array}\right]
$$

Now computing pair-wise matrix for alternatives

\section{For remote sensing image in terms of Image criteria}

\begin{tabular}{|l|l|l|l|l|l|}
\hline & DWT & PCA & IHS & Laplacian & Contrast \\
\hline DWT & 1 & 3 & 5 & 7 & 5 \\
\hline PCA & $1 / 3$ & 1 & 3 & 5 & 7 \\
\hline IHS & $1 / 5$ & $1 / 3$ & 1 & 5 & $1 / 3$ \\
\hline Laplacian & $1 / 7$ & $1 / 5$ & $1 / 5$ & 1 & $1 / 4$ \\
\hline Contrast & $1 / 5$ & $1 / 7$ & 3 & 4 & 1 \\
\hline
\end{tabular}

which can be represented in form of matrix as

$$
\left[\begin{array}{ccccc}
1 & 3 & 5 & 7 & 5 \\
0.33 & 1 & 3 & 5 & 7 \\
0.20 & 0.33 & 1 & 5 & 0.33 \\
0.14 & 0.20 & 0.20 & 1 & 0.25 \\
0.20 & 0.14 & 3 & 4 & 1
\end{array}\right]
$$


Signal \& Image Processing : An International Journal (SIPIJ) Vol.6, No.5, October 2015

Computing normalized eigenvector for above pair wise matrix we get,

$$
\left[\begin{array}{l}
0.4613 \\
0.2990 \\
0.0877 \\
0.0364 \\
0.1156
\end{array}\right]
$$

For remote sensing image in terms of sensors criteria

\begin{tabular}{|l|l|l|l|l|l|}
\hline & DWT & PCA & IHS & Laplacian & Contrast \\
\hline DWT & 1 & 5 & 3 & 7 & 9 \\
\hline PCA & $1 / 5$ & 1 & 7 & 5 & 9 \\
\hline IHS & $1 / 3$ & $1 / 7$ & 1 & 5 & $1 / 3$ \\
\hline Laplacian & $1 / 7$ & $1 / 5$ & $1 / 5$ & 1 & $1 / 4$ \\
\hline Contrast & $1 / 9$ & $1 / 9$ & 3 & 4 & 1 \\
\hline
\end{tabular}

which can be represented in form of matrix as

$$
\left[\begin{array}{ccccc}
1 & 5 & 3 & 7 & 9 \\
0.2 & 1 & 7 & 5 & 9 \\
0.33 & 0.14 & 1 & 5 & 0.33 \\
0.14 & 0.20 & 0.20 & 1 & 0.25 \\
0.11 & 0.11 & 3 & 4 & 1
\end{array}\right]
$$

Computing normalized eigenvector for above pair wise matrix we get,

$$
\left[\begin{array}{l}
0.5093 \\
0.3014 \\
0.0747 \\
0.0313 \\
0.0832
\end{array}\right]
$$

For remote sensing image in terms of application criteria

\begin{tabular}{|l|l|l|l|l|l|}
\hline & DWT & PCA & IHS & Laplacian & Contrast \\
\hline DWT & 1 & 4 & 6 & 5 & 7 \\
\hline PCA & $1 / 4$ & 1 & 7 & 5 & 9 \\
\hline IHS & $1 / 6$ & $1 / 7$ & 1 & 6 & $1 / 3$ \\
\hline Laplacian & $1 / 5$ & $1 / 5$ & $1 / 6$ & 1 & $1 / 4$ \\
\hline Contrast & $1 / 7$ & $1 / 9$ & 3 & 4 & 1 \\
\hline
\end{tabular}

which can be represented in form of matrix as

$$
\left[\begin{array}{ccccc}
1 & 4 & 6 & 5 & 7 \\
0.25 & 1 & 7 & 5 & 9 \\
0.17 & 0.14 & 1 & 6 & 0.33 \\
0.20 & 0.20 & 0.17 & 1 & 0.25 \\
0.14 & 0.11 & 3 & 4 & 1
\end{array}\right]
$$


Signal \& Image Processing : An International Journal (SIPIJ) Vol.6, No.5, October 2015

Computing normalized eigenvector for above pair wise matrix we get,

$$
\left[\begin{array}{l}
0.4841 \\
0.3146 \\
0.0732 \\
0.0372 \\
0.0909
\end{array}\right]
$$

$\underline{\text { For remote sensing image in terms of resource criteria }}$

\begin{tabular}{|l|l|l|l|l|l|}
\hline & DWT & PCA & IHS & Laplacian & Contrast \\
\hline DWT & 1 & 6 & 7 & 5 & 9 \\
\hline PCA & $1 / 6$ & 1 & 8 & 7 & 9 \\
\hline IHS & $1 / 7$ & $1 / 8$ & 1 & 6 & $1 / 3$ \\
\hline Laplacian & $1 / 5$ & $1 / 7$ & $1 / 6$ & 1 & $1 / 4$ \\
\hline Contrast & $1 / 9$ & $1 / 9$ & 3 & 4 & 1 \\
\hline
\end{tabular}

which can be represented in form of matrix as

$\left[\begin{array}{ccccc}1 & 6 & 7 & 5 & 9 \\ 0.17 & 1 & 8 & 7 & 9 \\ 0.14 & 0.13 & 1 & 6 & 0.33 \\ 0.20 & 0.14 & 0.17 & 1 & 0.25 \\ 0.11 & 0.11 & 3 & 4 & 1\end{array}\right]$

Computing normalized eigenvector for above pair wise matrix we get,

$$
\left[\begin{array}{l}
0.5463 \\
0.2792 \\
0.0625 \\
0.0331 \\
0.0762
\end{array}\right]
$$

In order to find finalized ranking, the eigenvectors of alternatives/image fusion techniques are concatenated into onto one matrix which multiplied by eigenvector of criteria as follows:

$$
\left[\begin{array}{llll}
0.4613 & 0.5093 & 0.4841 & 0.5463 \\
0.2990 & 0.3014 & 0.3146 & 0.2792 \\
0.0877 & 0.0747 & 0.0732 & 0.0625 \\
0.0364 & 0.0313 & 0.0372 & 0.0331 \\
0.1156 & 0.0832 & 0.0909 & 0.0762
\end{array}\right] *\left[\begin{array}{l}
0.1865 \\
0.1500 \\
0.4521 \\
0.2114
\end{array}\right]=\left[\begin{array}{l}
0.497 \\
0.302 \\
0.074 \\
0.035 \\
0.091
\end{array}\right]
$$

Hence final aggregated scores of all the image fusion techniques is :

$$
\left[\begin{array}{l}
0.497 \\
0.302 \\
0.074 \\
0.035 \\
0.091
\end{array}\right]
$$


Signal \& Image Processing : An International Journal (SIPIJ) Vol.6, No.5, October 2015

The finalized score suggest following ranking :

\begin{tabular}{|c|c|}
\hline Ranking & Image fusion Technique \\
\hline 1 & DWT \\
\hline 2 & PCA \\
\hline 3 & Contrast \\
\hline 4 & IHS \\
\hline 5 & Laplacian \\
\hline
\end{tabular}

\section{CONCLUSions}

We proposed a novel approach for automating the process of image fusion using multi-criteria decision making based on Analytical Hierarchical Process. The aim of this approach is to provide most accurate selection of fusion algorithms along with adaptive learning of the system with the help of knowledge base. The system is expected to work well in most scenarios and does resolve problems earlier faced by the systems with manual selection process.

\section{REFERENCES}

[1] T.L. Saaty, "Relative Measurement and its Generalization in Decision Making: Why Pair Wise Comparisons are Central in Mathematics for the Measurement of Intangible Factors - The Analytic Hierarchy/Network Process". Review of the Royal Spanish Academy of Sciences, Series A, Mathematics vol. 102 , 2, December 2007, pp 251-318.

[2] The Analytic Hierarchy Process, http://www.dii.unisi.it/ mocenni/Note_AHP.pdf.

[3] Analytic Hierarchy Process, http://en.wikipedia.org/wiki/Analytic_hierarchy_process.

[4] An Illustrated Guide to the Analytic Hierarchy Process, https://mi.boku.ac.at/ahp/ahptutorial.pdf.

[5] E. Triantaphyllou and S Mann, "Using the Analytic Hierarchy Process for Decision Making in Engineering Applications: Some Challenges", http://bit.csc.lsu.edu/trianta/Journal_PAPERS1/AHPapls1.pdf.

[6] Analytic Hierarchy Process, http://www.dept.aoe.vt.edu/ cdhall/courses/aoe4065/AHPslides.pdf.

[7] T. Saaty, "Decision Making with the Analytic Hierarchy Process", "http://citeseerx.ist.psu.edu/viewdoc/download?doi=10.1.1.409.3124\&rep=rep1\&type=pdf.

[8] M. Alexander, "Decision-Making using the Analytic Hierarchy Process and SA/IML", http://analytics.ncsu.edu/sesug/2012/SD-04.pdf.

[9] https://www.scss.tcd.ie/Rozenn.Dahyot/CS1BA1/SolutionEigen.pdf.

[10] Elizabeth Diaz, Arijit De, "Search Engine Result Aggregation Using Analytical Hierarchy Process", WI-IAT, 2010, Web Intelligence and Intelligent Agent Technology, IEEE/WIC/ACM International Conference on, Web Intelligence and Intelligent Agent Technology, IEEE/WIC/ACM International Conference on 2010, pp. 300-303.

[11] Finding Eigenvalues and Eignvectors, https://www.scss.tcd.ie/Rozenn.Dahyot/CS1BA1/SolutionEigen.pdf.

[12] P. Dehghanian, .A. Razi Kazemi,.M. , "Optimal RTU placement in power distribution systems using a novel method based on analytical hierarchical process (AHP)", 10th International Conference on Environment and Electrical Engineering, 2011, pp. 1-4.

[13] F. Ahmad et al., "A framework of integrated decision support system for students' projects evaluation", 2012 International Conference on Information Retrieval and Knowledge Management, pp. 82-86.

[14] Haibin Yuan, "Multi-agent Approach for Monitoring and Diagnosis Evaluation of Electric Apparatus Control System", 2006 Mechatronics and Automation Conference, pp. 815-819.

[15] R. Kant et al., " Knowledge management implementation: Analytic hierarchy process methodology", 2011 Industrial Engineering and Engineering Management Conference, pp. 1033-1037. 
Signal \& Image Processing : An International Journal (SIPIJ) Vol.6, No.5, October 2015

[16] Muhammad Atif Iqbal et al., "A New Requirement Prioritization Model for Market Driven Products Using Analytical Hierarchical Process ", 2010 Conference on Data Storage and Data Engineering, pp. 142-149.

[17] A. Kumar et al., "Multi-agent Based Intelligent System for Image Fusion", Advances in Computational Science, Engineering and Information Technology, Springer, vol. 225, pp. 101-110.

[18] H. Yuan, "A novel method for image feature fusion based on MADM", 2011 Conference on Multimedia Technology, pp. 2877-2879.

[19] Firouz Abdullah Alwassai et al., "The IHS Transformations Based Image Fusion ", International Journal of Advanced Research in Computer Science, vol. 2, number 5, 2011.

[20] A. Singh et al., "Implementation \& comparative study of different fusion techniques (WAVELET, IHS, PCA)", International Journal of Engineering and Science, vol. 1, issue 4, 2012.

[21] Mohamed R. Metwalli et al., "Image Fusion Based on Principal Component Analysis and High-Pass Filter", 2009 Conference on Computer Engineering and Systems, pp. 63-70.

[22] S. Krishnamoorthy et al., "Implementation and Comparative Study of Image Fusion Algorithms", International Journal of Computer Applications, 2010.

[23] K. Rani et al., "Study of Different Image fusion Algorithm", IJETAE, vol. 3, issue 5, 2013.

[24] Hamid Sheikh et al., "Image Information and Visual Quality", http://live.ece.utexas.edu/publications/2004/hrs_ieeetip_2004_imginfo.pdf.

[25] C.S. Xydeas and V. Petrovic, "Objective image fusion performance measure", Electronics Letters, vol. 36, number 4, 2000.

[26] B. Dey et al., "Assessment of Ph.D (Technology) quality in Indian universities: AHP based decision model", 2013 Conference on Teaching, Assessment and Learning for Engineering, pp. 484-489.

\section{AUTHORS}

Dr. Ashok Kumar is an Associate Professor of Computer Science and the Associate Director of the School of Computing and Informatics at the University of Louisiana at Lafayette. He obtained his bachelor's degree in computer science and engineering from the Indian Institute of Technology, Banaras Hindu University, and masters and Ph.D. in computer engineering from the University of Louisiana at Lafayette. He has published extensively and served on the organizing committees of several conferences in computing. He also serves on the editorial board of several peer reviewed journals.

Amruta Shelar received her masters degree in computer science from the University of Louisiana at Lafayette. She has extensive experience in Java and C\# based applications. She is working with Fareportal Inc. in New York city as Front End Engineer and making significant contributions to user experience of Fareportal's commercial apps and other products.

Dr. Jim Etheredge received the M.S. degree in computer science from the University of Southwestern Louisiana in 1986 and the Ph.D. in computer science from the University of Southwestern Louisiana in 1989. He is a professor of computer science at the University of Louisiana at Lafayette, Lafayette, LA and the coordinator for the Video Game Design and Development concentration of the undergraduate computer science curriculum. His research and teaching interests include video game design and development, artificial intelligence, multi-agent game systems, and database management systems
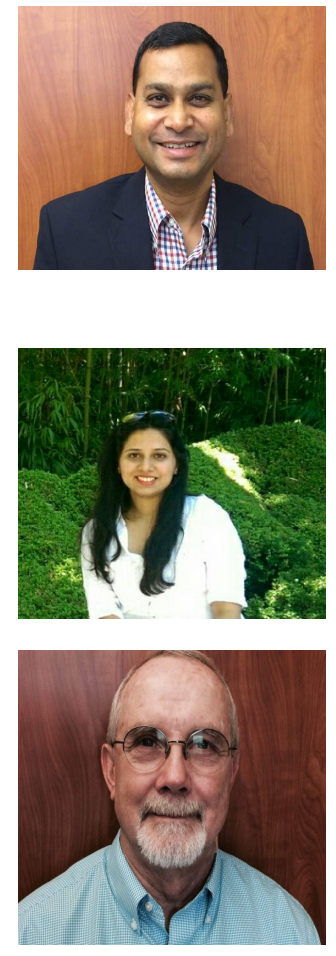
Signal \& Image Processing : An International Journal (SIPIJ) Vol.6, No.5, October 2015

Sandhya Prakash received her masters degree in computer application from Indira Gandhi National Open University, Delhi, India in 2011. She is interested in software engineering (especially for web based, enterprise systems, and business intelligence based systems) algorithms, and database management systems. She is experienced in developing Java Enterprise Applications and implementing performance enhancement measures in complex business. She has also worked with popular social media based APIs such as Gmail, Facebook, Linkedin, HotMail, Windows Live AOL, and Google. She is experienced in implementing SOAPful and RESTful web services.

Dr. Sudhansh Sharma is an Assistant Professor in the School of Computer and Information Sciences at the Indira Gandhi National Open University, India. He received his masters degrees in physics and computer science, a MBA degree in operations research, and a Ph.D. degree in physics. He has over 15 years on academic and industrial research experience.

He has a dozen of publications in various national and international journals, and serves on the editorial board of Global Journal of Enterprise Information Systems, an International Journal. He has authored a book entitled "Modeling Of Novel

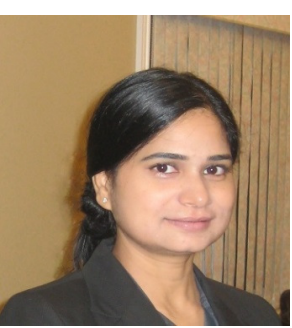
MOSFET Devices - Basics, Concepts, Methods", published by Lambert Academic Publishing (Germany).

M.P.Mishra is an Assistant Professor in School of Computer and Information Sciences, Indira Gandhi National Open University (IGNOU), New Delhi, India. He obtained bachelors and masters degrees with honors in computer science from Banaras Hindu University, and another masters degree in computer science from Jawaharlal Nehru University. Previously he worked as an Assistant Professor in IMT Ghaziabad before joining IGNOU. He was awarded University Silver Medal for best research paper in 2013 by IGNOU. He received the Certificate of Competence from IIT Kanpur and COL for "Mobile for Development, a Massive

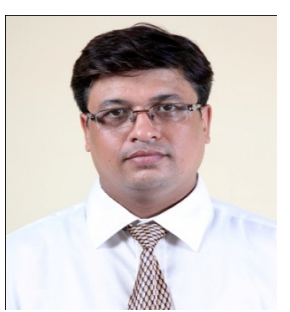
Open Online Course (MOOC) for Development", in December 2013. His area of interests includes object oriented technologies and enterprise applications, wireless communication and e-Learning. He is an active researcher and also takes a keen interest in educating his students. 